\title{
1 What is cost-efficient phenotyping? Optimizing costs for different scenarios
}

2 Daniel Reynolds ${ }^{+1}$, Frederic Baret ${ }^{+2}$, Claude Welcker $^{+3}$, Aaron Bostrom $^{+1}$, Joshua Ball ${ }^{1}$,

3 Francesco Cellini ${ }^{4}$, Argelia Lorence ${ }^{5}$, Aakash Chawade $^{6}$, Mehdi Khafif $^{7}, K_{0 j i}$ Noshita $^{8}$, 4 Mark Mueller-Linow ${ }^{9}$, Ji Zhou*1,10, François Tardieu*3

5

$6 \quad{ }^{1}$ Earlham Institute, Norwich Research Park, Norwich UK, NR4 7UH

$7 \quad 2$ INRA EMMAH - CAPTE, address, Avignon France

$8{ }^{3}$ INRA Univ Montpellier, LEPSE, 2 place Viala 34060 Montpellier France

$9 \quad{ }^{4}$ Agenzia Lucana di Sviluppo e di Innovazione in Agricoltura 75010 Metaponto (MT)

10 Italy

$11{ }^{5}$ Phenomics Facility, Arkansas Biosciences Institute, Arkansas State University, Jonesboro,

12 Arkansas, USA

$13{ }^{6}$ Department of Plant Breeding, Swedish University of Agricultural Sciences (SLU), P.O. Box 101, 1423053 Alnarp, Sweden

157 Université de Toulouse, INRA, CNRS, LIPM Castanet-Tolosan, France.

$16{ }^{8}$ Japan Science and Technology Agency (JST) Precursory Research for Embryonic Science and

17 Technology (PRESTO), Graduate School of Agriculture and Life Science, The University of Tokyo

189 Institute of Bio- and Geosciences (IBG), IBG-2: Plant Sciences, Forschungszentrum Juelich

$19 \mathrm{GmbH}$, Juelich, Germany

$20{ }^{10}$ Plant Phenomics Research Center, Nanjing Agricultural University, Nanjing China, 210095

$22+$ Joint first authors

$23{ }^{*}$ Corresponding Authors:

24 francois.tardieu@inra.fr, ji.zhou@earlham.ac.uk or ji.zhou@njau.edu.cn 
Abstract

Progress in remote sensing and robotic technologies decreases the hardware costs of phenotyping. Here, we first review cost-effective imaging devices and environmental sensors, and present a trade-off between investment and manpower costs. We then discuss the structure of costs in various real-world scenarios. Hand-held low-cost sensors are suitable for quick and infrequent plant diagnostic measurements. In experiments for genetic or agronomic analyses, (i) major costs arise from plant handling and manpower; (ii) the total costs per pot/microplot are similar in robotized platform or field experiments with drones, hand-held or robotized ground vehicles; (iii) the cost of vehicles carrying sensors represents only $5-26 \%$ of the total costs. These conclusions depend on the context, in particular for labor cost, the quantitative demand of phenotyping and the number of days available for phenotypic measurements due to climatic constraints. Data analysis represents $10-20 \%$ of total cost if pipelines have already been developed. A trade-off exists between the initial high cost of pipeline development and labor cost of manual operations. Overall, depending on the context and objectives, "cost-effective" phenotyping may involve either low investment ("affordable phenotyping"), or initial high investments in sensors, vehicles and pipelines that result in higher quality and lower operational 41 costs.

\section{Highlights}

43 - New technologies considerably reduce the costs of sensors and automated vehicles

44 - Low investment in sensors, vehicles or pipelines present trade-offs with labor costs

45 - Plant/plot handling and labor costs represent the major proportion of costs in phenotyping 46 experiments

47 - The costs of high-throughput experiments in the field and in automated platforms is similar 48 regardless of vehicles

49 - The development of software applications (e.g. imaging, phenotypic analyses, models, 50 information system) is a major part of costs

51 Keywords Phenotyping; Phenomics; Cost; imaging; information system; affordable; 
53 Table of contents

54 Introduction

55 I Imaging techniques with a range of hardware costs

$56 \quad 1.1$ Handheld phenotyping technologies

$57 \quad 1.2$ Aerial imaging for large-scale phenotyping

$58 \quad 1.3$ Imaging with ground vehicles

$59 \quad$ 1.4 Environmental measurements

60 II Costs associated with image capture represent a fraction of the overall cost of phenotyping

$61 \quad 2.1$ A method for calculating costs in field and greenhouse platforms

$62 \quad 2.2 \mathrm{~A}$ high cost for plant management

632.3 Investing in appropriate environmental characterization results in comparatively low cost 64 for a high return

$65 \quad 2.4$ Imaging costs: a trade-off between investment and labor costs

2.4.1 The choice of vehicle mostly depends on the demand for microplots per year

2.4.2 The cost of imaging devices is similar to that of vehicles that carry sensors

2.5 Costs of typical experiments

69 2.5.1 Image analysis: a tradeoff between investment in automated workflows and day-to-day

$70 \quad$ labor costs

$71 \quad$ 2.5.2 High costs for data analysis for the identification of traits

72 2.5.3 Costs associated with data storage and organization ensure the possibility of reusing $73 \quad$ datasets

74 III An unexpected structure of costs has large consequences on conclusions about cost 75 effectiveness 
The observation of growing plants can involve operations of different nature. For instance, when a farmer visits fields to decide if and when an operation needs to be carried out, e.g. irrigation, fertilization or harvest, this is essentially based on direct observations that may be helped by lowthroughput tools. The same tools can be used in nurseries when a breeder rapidly inspects tens of thousands of plants of a population with the aim of identifying, for instance, plants of abnormal aspect or with high sensitivity to a disease. At the other extreme, genome wide association studies (GWAS) or genomic predictions require analysis of hundreds of lines to identify the genetic variability of traits associated with plant performance in diverse conditions. This translates into thousands of plants in greenhouse robotized platforms, or of microplots (i.e. a plot of typically 4-10 $\mathrm{m}^{2}$ with a single genotype) in field experiments. Such experiments involve (i) novel technologies for collecting relevant images of each plant or microplot, able to characterize the temporal and spatial variability of traits; (ii) the design and maintenance of pipelines of image analyses allowing one to extract quantitative traits from images; (iii) analyses of datasets originating from different installations at different scales (e.g. phenotyping platforms in greenhouses or in the field at organ, plant or canopy levels); and (iv) shared information systems able to manage and store data in such a way that data can be re-used or re-analyzed by the scientific community [1-3].

95 The concept of "affordable phenotyping" or "cost-effective phenotyping" has developed rapidly in recent years due to decreasing cost of equipment such as low-cost environmental sensors [4] or smartphone-embedded and mobile imaging sensors [5]. Indeed, cost-effective phenotyping approaches have been utilized to capture image- and sensor-based crop performance datasets in greenhouses and in the field [6-8]. For example, ground-based portable devices $[9,10]$ have been used to estimate canopy photosynthesis rate at key developmental stages; mobile phone cameras are also used to capture crop disease symptoms and plant morphology [11-15]; unmanned aerial vehicles (UAVs) equipped with relatively low-cost RGB (red-green-blue) 104 cameras are employed to study crop performance and field variability under different growing conditions [16-18]. 
105 Depending on the number and complexity of operations associated with the observation of a 106 given set of phenotypic traits, the cost of equipment can represent a variable fraction of the total 107 cost of the phenotyping program. Hence, the cost of specific pieces of equipment should be 108 considered as a part of the costs of the whole phenotyping process. For example, low-cost 109 hardware can be appropriate for diagnostic or quick characterization of a few plants in a field 110 experiment. If many plants or plots have to be sampled several times during the crop cycle, this 111 may result in higher cost related to the additional human effort required for the analysis of poorly 112 calibrated and documented data, in order to obtain interpretable and heritable variables.

113 Plant breeding programs are also potential end-users of phenomics and need to analyze whether 114 the investment in a particular phenotyping technology will achieve a justifiable increase in the 115 rate of genetic gain. It is important to acknowledge here that, at this stage, the extent to which 116 phenomics can substantially increase this rate is discussed. Breeders have been successful in 117 increasing yield, e.g. in wheat $[19,20]$ and maize [21], essentially based on direct selection for 118 yield. The success of trait-based selection has been focused on visually observable traits such as 119 anthesis-silking interval in maize, disease symptoms, growth phenotypes [22], and flowering [23], 120 which do not require high investment. Novel breeding techniques such as genomic selection may 121 reinforce the power of yield-based selection perhaps at the expense of trait-based selection $122[20,24]$, thereby decreasing the interest of phenotypic analyses to focus on increasing the 123 average yield in a given region [20]. It has been proposed that the contribution of phenomics to 124 pre-breeding may involve novel biological applications, for instance (i) where and when do genotypes or alleles present in the genetic diversity present comparative advantages, and (ii) 126 whether one can make the best use of combinations of alleles controlling adaptive traits (e.g. the 127 controls of stomatal conductance or growth) as a function of environmental conditions [25]. 128 These questions involve a combination of phenomics, modelling and genomic prediction to 129 assess the genetic and environmental controls of plant adaptation [25]. Addressing the above 130 questions may be essential for breeding in a context of climate change, but it is currently 131 upstream of most breeding programs. Until clear contributions of phenomics to breeding have 132 been demonstrated in particular contexts, it might be misleading to attempt to evaluate the 
133 efficiency of phenomics techniques, either 'envirotyping' or plant measurements, in terms of cost 134 per unit genetic gain.

135 Hence, we hereby focus on the costs of all operations involved in phenomics, and not on the 136 efficiency of their costs for breeding. We first review the current imaging techniques and vehicles 137 carrying the corresponding sensors. We then present the structure of costs associated with 138 phenomics based on case studies for different experiments in the field or in indoor controlled 139 conditions and for different imaging systems.

140 I Imaging techniques with a range of hardware costs

\section{$141 \quad 1.1$ Handheld phenotyping technologies}

142 Small, lightweight and reusable devices considerably reduce the hardware costs associated with 143 handheld phenotyping at canopy or leaf level in field conditions, but also at plant level in indoor 144 conditions. For example, using an advanced software approach and commercially available 145 handheld digital cameras, 3D reconstructions at organ level can either be accomplished by 146 combining tens of images of a single plant taken by hand with structure-from-motion and multi147 view stereo techniques [26] or by using stereo camera setups and stereo image processing [27]. 148 A 3D reconstruction of a plant row has been performed using a bespoke hand-held sensor 149 platform [28], while a standard RGB camera was used to record color information of scanned 150 areas. A visual-inertia and 2D LiDAR (Light Detection and Ranging) sensor contributed to the 151 reconstruction of colored 3D models of crop areas. Another device connects infrared 152 temperature sensing, GPS positioning and a normalized difference vegetation index (NDVI) 153 sensor, together with a standard laptop mounted to a hand-held pole [29]. A handheld device 154 combines light-emitting diode (LED) lights with visible and infrared sensors in a package able to 155 calculate light transmission through the surface of a leaf, fluorescence-based kinetics and 156 photosynthesis-associated variables [30]. Standard RGB cameras have been widely used to 157 characterize the canopy structure [31,32], with adaptation to smartphone cameras [15].

158 The phenotyping devices described above present several limitations. Lower investment costs 159 are most often at the expense of labor-intensive manual control and analysis, otherwise they 160 may lead to the production of non-repeatable datasets. Indeed, these approaches require human 
161 decisions for the imaged area, the selection of regions of interest, and, finally, analytical software

162 to standardize and analyze the captured data [5]. Furthermore, the scale of measurement is

163 limited without costly and complex machinery. Hence, it can be considered that handheld devices

164 are most appropriate for actions with limited throughput carried out by experienced plant 165 specialists

\subsection{Aerial imaging for large-scale phenotyping}

167 Aerial imagery for field conditions provides a sufficient throughput to sample all the plots of a 168 field experiment (typically thousands of microplots) within a short time interval. It is efficient 169 when targeting canopy characteristics that may vary considerably within a short time interval 170 such as canopy temperature [33,34] or changes in canopy structure due to leaf rolling [35].

171 Traditional manned helicopters are still used because of the heavy payload capacity [36].

172 Nevertheless, three factors have triggered the rapid development of UAVs for field phenotyping 173 applications in the last five years: (i) the increasing autonomy reliability and payload capacity, (ii) 174 the decrease of the corresponding cost, together with an increase in sensor performance, and 175 (iii) the development of image processing software allowing to precisely compute the position of 176 the UAV corresponding to each individual image and to create an orthomosaic image map of the 177 field [17]. The high-resolution imagery provided by consumer grade RGB cameras has been used 178 to count plants and organs [37] and to evaluate the cover fraction [38]. Using the same RGB cameras, the shape from motion algorithm creates the dense 3D point clouds from which plant

180 height is derived with a very good accuracy [39-42]. Light-weight LiDAR was also tentatively 181 mounted on UAVs to get a more direct estimation of plant height and canopy related traits [43].

182 Multispectral and hyperspectral images were used to assess canopy characteristics including the 183 green area index [44] and canopy temperature [34].

184 The high-throughput of UAV-based observations and its relatively affordable cost makes it 185 potentially very efficient for field phenotyping. However, it needs to operate under favorable 186 conditions, i.e. with no rain, when the illumination is relatively stable and when the wind is not 187 too strong (typically wind speed lower than $35 \mathrm{~km} \mathrm{~h}^{-1}$ ). This limits the proportion of days during 188 which this technique can be used, thereby increasing the cost per day (see Section II). 
189 Furthermore, the massive number of images produced and the intensive computation required

190 to accurately locate images and extract the corresponding microplots contributes to the 191 significant increase of the cost of the traits analyses derived from this technique. Except for LiDAR 192 techniques, the passive nature of UAV observations (the sun being the unique light source) makes 193 the quantification of traits prone to biases due to the specific illumination conditions at the time 194 of image acquisition [45,46]. Recently, UAV costs have increased due to legislation and training 195 issues (see section II)

\subsection{Imaging with ground vehicles}

197 Low-cost mobile phenotyping systems have been developed by attaching imaging components 198 to existing farm equipment. For example, a tractor can pull a trailer equipped with sensors 199 including a color camera, multiple laser distance scanners, and a hyperspectral imaging sensor $200[6,46]$. Simpler moveable carts have been designed to reduce costs by not requiring pre-existing 201 agricultural equipment, but this is limited to crops with relatively low plant height $[47,48]$. 202 Alternatively, a large sealed box has been placed around individual plots to capture multi-spectral 203 measurements [49]. A standalone manned vehicle has been developed to carry a thermal 204 infrared camera and a low-cost LiDAR together with light riggings and height adjustable 205 mechanism [4]. Similarly, a mobile phenotyping platform has been developed, equipped with 206 fully adjustable and swappable sensors [29].

207 The hidden costs of using the phenotyping devices presented above are data calibration, data 208 management and processing. Calibrating the data captured by sensors with manned vehicles in 209 the field can be a time-consuming task due to wide variations in different sensor groups as well 210 as field regions. It is not only costly but also technically complex to consistently store large 211 quantities of images and sensor data throughout the growing season and associating important 212 metadata (e.g. a time stamp and the corresponding spatial coordinates). Furthermore, well213 trained, thus expensive, specialists are needed to operate these manned phenotyping devices.

214 As a result, more expensive autonomous robotic vehicles have been developed [50]. For example, 215 a fully automatic unmanned robot was specifically designed for field phenotyping applications, 216 controlled by an RTK-GPS positioning system with centimeter accuracy and equipped with 
217 modular sensors including LiDAR, multispectral cameras and high resolution RGB cameras [47].

218 Other vehicles can collect images in a field, together with performing tasks such as seeding,

219 weeding, and harvesting [51]. A robot system has been used to image and analyze berry structure

220 and color in grapevine breeding [48]. Robots with specific phenotyping tasks have also been

221 developed to work alongside a static tower system [52]. Such robotic solutions offer the capacity

222 to use artificial illumination (active imaging), independent from natural illumination conditions

223 (even during the night or cloudy days).

\section{$224 \quad 1.4$ Environmental characterization and envirotyping}

225 Weather stations with data loggers are now widely available for a much reasonable price, thereby 226 making hourly environmental characterization a routine procedure. This can be extended to 227 additional measurements such as soil water content/potential and soil temperature. For 228 instance, electronic tensiometers have been deployed in a network of field experiments for a 229 limited cost [53]. The same applies to installations in controlled conditions, for which 230 measurements of local environmental conditions can be performed with a time step of minutes 231 [54]. Using an "open hardware" design strategy, soil moisture data loggers have been produced 232 using commercially available electronics and sensors [7]. Usability is increased by data 233 transmission over General Packet Radio Service (GPRS), allowing results to be collated off-site 234 without manual harvesting. In addition to GPRS, radio transmission can also be used for data 235 communication within a more complicated network of modular devices [55].

236 A specific sampling strategy is required to represent the spatial variability of environmental 237 conditions in the field while using fixed sensors. Another problem is the software R\&D costs to 238 cross-reference different static devices in order to extract meaningful information from collected 239 crop image series and climate datasets using advanced computer vision and data analytic 240 packages [53]. Small workstations have been developed to provide plot level crop growth traits 241 as well as micro-environment variables [56]. Multiple sensor types can be integrated into single242 board computers that can then form a scalable, multi-point in-field network to assist decision 243 making processes such as crop management and line selection. Modelling is another efficient 244 method for assessing the spatial variability of environmental conditions, in particular in 
245 greenhouse platforms, thereby limiting the number of environmental sensors deployed in 246 experiments [54].

247 To our knowledge, the use of sensor networks is currently the main contribution of phenomics 248 to plant breeding, via the development of 'envirotyping' [57-59]. It has been increasingly used 249 by breeding companies for the identification of environmental scenarios in which combinations 250 of alleles have positive effects on yield [24,53], the identification of target populations of 251 environments associated with a breeding program [24,60], or even the definition of new criteria 252 for developing commercial makes of resilient genotypes [61].

253 II Costs associated with image capture represent a limited fraction of the overall 254 cost of phenotyping

\subsection{A method for calculating costs in field and greenhouse platforms}

256 Calculating costs with a consistent method for field and platform phenotyping is a challenging 257 task because it is associated with hypotheses and simplification that are debatable by nature. In 258 Tables 1 and 2, examples for calculation of costs are shown in the field with either automated 259 ground vehicle, a hand-driven ground vehicle (e.g. handcart or wheelbarrow style trolleys) or a 260 UAV, or in controlled conditions with a robotized phenotyping platform. Table 1 presents costs 261 associated with imaging for the typical number of plants or microplots in experiments for each 262 technique, under two scenarios: (i) in the 'offer limited' scenario, the use of devices is limited by 263 the availability of equipment or personnel; (ii) in the 'demand limited' scenario, it is limited by 264 the number of applications for experiments by public or private users. Both scenarios can co265 exist, for example between years depending on the amount of available funding for Plant Science, 266 or between installations depending on the demand at a given time. Table 2 presents all costs 267 associated with a typical experiment using methods presented in Table 1 in the two above 268 scenarios, including costs for infrastructure, data management and data storage. Both tables 269 result from surveys performed in the French phenotyping infrastructure Phenome-EMPHASIS.fr 270 project (www.phenome-EMPHASIS.fr), weighted with information generated from other 
271 infrastructures in UK, USA and Germany. It is noteworthy that these costs correspond to

272 countries where the labor cost is high. Hence, the conclusions of this study need to be 273 contextualized.

274 In field experiments, the cost for imaging (e.g. vector and sensors) was calculated over the whole 275 lifetime of the considered device, taking into account the number of imaged plots per year 276 (number of days of use per year $x$ number of plots measured every day), and the expected 277 lifetime of the considered device (in years). The investment cost is therefore expressed per 278 plot.day per year. The number of days of use per year differs between techniques, and varies 279 between sites with the frequency of weather limitations. For instance, this number is higher for 280 automated ground vehicles with active imaging assisted with artificial light (which can be used 281 even in very cloudy or night conditions) than for hand-held ground vehicles (limited by light 282 intensity because of passive imaging) and UAVs (limited by weather constraints, in particular 283 wind, rain, and light because of passive imaging). This results in costs relative to that of the 284 automated ground vehicle of 1.00, 0.83 and 0.67 , respectively for an automated ground vehicle, 285 a hand-held ground vehicle and a UAV (Table 1). The costs also depend on the local demand for 286 the selected device: the investment cost per plot.day per year was calculated as higher if the use 287 of the device was limited due to low demand (scenario 2 in Table 1 ) than if the device was used 288 at full capacity (scenario 1). Additionally, the calculations shown in Table 1 also depend on the 289 expected lifetime of the considered device, which is higher for a ground vehicle than for a UAV. 290 Sensors were considered as having a shorter expected duration than vehicles because of 291 obsolescence. The labor cost was calculated by dividing the annual cost (220 working days per 292 year) by the number of days required for the considered operation and the number of microplots 293 to be sampled per year. The same calculations were considered for a robotized platform, 294 expressed per plant.day. In the case presented in Table 1, the platform was considered as being used in three experiments per year, with a 90-day duration each.

296 The above information was then used for calculating the cost of a typical experiment (Table 2), 297 either in a field platform with 1,700 microplots (e.g. 284 genotypes, 2 treatments and 3 298 replicates) and 10 days of measurement to monitor the crop cycle, or in a platform with 1,700 299 plants over 90 days. The costs for plant handling, for image capture, image analysis, data analysis 
300 itself and data storage considered in the analysis are presented in Table 2. Data in Tables 1 and 2

301 are presented below.

$302 \quad 2.2$ A high cost for plant management

303 Phenotyping is, by definition, associated with a field, a greenhouse or a growth chamber in which

304 experiments are carried out. Field phenotyping involves a cost of typically $\$ 30$ to $\$ 50$ USD per

305 microplot for one experiment, resulting in \$68K USD for a typical experiment involving 1,700

306 microplots necessary for genetic analyses (Table 2). This price is used internally or externally by

307 many breeding companies and includes the cost of hiring the field, plant management, irrigation

308 and harvest. Greenhouse experiments are also expensive, with a typical investment of one

309 million dollars for a greenhouse equipped with climatic control and surrounding facilities allowing

310 compost management, potting and cleaning. Another million is required for the robots

311 associated with the handling of the thousands of plants involved in genetic analyses, including

312 imaging cabins, watering and weighing stations and conveyors. With the hypothesis of a given

313 equipment used for 15 years with three experiments per year, this investment results in a cost

314 of \$67K USD for an experiment handling 1,700 plants, to which one adds a cost of \$5K USD for

315 electricity and potting compost. The cost per unit sample (microplot or plant) is therefore similar

316 to experiments either in the field or in a robotized platform (Table 2). Interestingly, some

317 platforms are in open air [62], thereby avoiding the cost of a greenhouse. This considerably

318 decreases experimental costs, provided that climatic conditions at the dedicated site allow

319 several experiments per year in open air; otherwise this approach could result in a high cost per

320 experiment if only one experiment can be accomplished per year. Overall, the high price per

321 microplot in the field or per plant in the greenhouse suggests that phenotyping experiments are

322 expensive per se before any phenotypic analyses are carried out.

2.3 Investing in an appropriate environmental characterization results in comparatively low cost for a high return.

325 The cost of environmental sensors has decreased rapidly (see section 1.4): climate sensors for 326 temperature and humidity normally cost less than \$5 USD per unit. Commercial devices can 327 provide, for a few thousand dollars, hourly measurements of the main environmental variables 
necessary to characterize an experiment site, including light, air temperature, relative humidity, rainfall, and wind speed. Soil water potential can also be characterized for a few hundred dollars with tensiometers, and soil water content for a few thousand dollars with capacitive sensors. In the calculations presented in Tables 1 and 2, this investment results in a cost of less than \$10K USD per field, with an assumption that the installed devices can last for about four years. An appropriate environmental characterization is therefore a cheap investment compared with plant management. Importantly, it allows joint analyses of several experiments both in the field and greenhouse, thereby improving one's ability to analyze datasets based on environmental scenarios or regression analyses $[53,63]$. Most breeding companies have now invested in this domain. Their feedback (personal communication), consistent with our perception, is that the major cost associated with environmental characterization is manpower because sensors have to be installed, then checked regularly and datasets need to be collected and then analyzed by semi-automated methods. In particular, for detection of outlier dates or sites, extra human costs are inevitable when many sensors are deployed under natural conditions.

\subsection{Imaging costs: a trade-off between investment and labor costs}

343 Imaging costs reported in Table 1 include the cost of the vector (e.g. manual measurements, UAV, ground vehicles), imaging hardware and associated software. These costs can range from a few dollars, in case of a person carrying a cell phone equipped with an imaging software, to hundreds of thousand dollars for a fully-equipped ground vehicle.

\subsubsection{The choice of a vehicle mostly depends on the demand for microplots per year.}

348 Portable devices have shown their ability to collect plant images in the field but their throughput

349 is low and they require experienced specialists (see Section 1.1). This limits their application to 350 relatively infrequent phenotyping for decision making or characterization of outlier genotypes.

351 We have therefore not considered them in the calculations of Tables 1 and 2, because they respond to a different use in relation to the costs associated with high-throughput phenotyping. UAVs are relatively cheap (a few thousand dollars) and can cover typically 4,000 microplots per day in 2-3 flights, resulting in a low-cost investment per plot.day. However, their expected 
and cloud coverage. Significant costs for insurance may occur in some countries. The manpower costs may be high in some countries due to civil aviation rules requiring authorizations and permits, leading to a cost of tens of thousands of dollars for training at least three persons per site. A calculation based on a throughput of 4,000 microplots per day, 40 available days per year, a lifetime of two years and personnel costs, still results in the lower cost compared with other vehicles (\$0.29 USD per plot.day per year, scenario 1 Table 1). This cost is increased to \$0.98 USD per plot.day per year in case of a lower demand of only 4,000 microplots per year (scenario 2, Table 1).

364 Hand-held ground vehicles have a cost of a few ten thousand dollars, excluding sensors. They can reach a throughput of around 100 microplots per hour. However, this approach struggles if 366 aiming at measuring thousands of plots with high frequency. Indeed, it requires well-trained 367 personnel who can manage the device, but who also accepts to push it for weeks during key 368 developmental stages, sometimes in bad weather conditions. This can cause difficulties in the management of the personnel. We have considered a throughput of 800 microplots per day over 50 days per year, which is probably a maximum in many countries but can be extended in others. The corresponding cost in Table 1 is \$0.98 USD per microplot.day per year. This cost is valid in the two hypotheses for demand in Table 1, because this method is associated with a lower throughput than UAVs.

374 Automated ground vehicles can be used over a larger number of days than hand-held ground 375 vehicles and UAVs, calculated as 60 days per year in Table 1 (5 months, 12 days per month). This 376 can be increased in case of fully automated vehicles equipped with active imaging (with 377 autonomous lighting), which allows their use in any conditions including during the night. Their 378 investment cost is high and essentially depends on the plant species used in experiments. For 379 instance, a vehicle allowing imaging cereal crops with $60 \mathrm{~cm}$ height grown in rows can lead to an 380 investment of typically $\$ 300 K$ USD, but the investment increases if the vehicle must also be used 381 for phenotyping tall species such as sorghum or maize, and/or crops that are not grown in rows 382 such as canola (typically \$500K USD). Taking into account the total investment, a throughput of 3831,200 microplots per day, a lifetime of 20 years and the personnel costs, the cost is $\$ 1.02$ USD 
384 per microplot.day per year in scenario 1 with a fully occupied usage, but will increase to $\$ 1.67$

385 USD per microplot.day per year in scenario 2 with a limited demand.

386 Hence, the investment cost corresponding to vectors largely depends on the use of the chosen

387 vector. For instance, robotized and hand-held ground vehicles result in similar costs if they are 388 used to their maximum potential (i.e. a high demand), whereas the robotized ground vehicle is 389 the most expensive option in a scenario with a limited demand. Similarly, UAVs appear to be a 390 low-cost option in the scenario with a high demand, whereas costs of UAVs and ground vehicles 391 are higher with a lower demand. An alternative solution for UAVs might be to rely on specialized 392 companies that carry out measurements. However, the economic models for such services in 393 phenotyping experiments are not yet stabilized.

$394 \quad$ 2.4.2 The cost of imaging devices is similar to those of vehicles that carry sensors

395 The costs of cameras (several hundred dollars per unit), portable multi-spectral devices (\$5-10K 396 USD), and mobile LiDAR (\$10-200K USD, depending on the resolution) are also high. The lifespan 397 of multi-spectral sensors and LiDAR can be several years, but they have been limited to four years 398 in Table 1 because of obsolescence. Personnel costs result in around \$0.2-0.4 USD per 399 microplot.day in European conditions, which can vary due to the frequency of phenotyping, 400 selected imaging sensors, and associated training costs. On these bases, the cost of imaging was 401 similar to ground vehicles, but much higher than UAVs.

\subsection{Costs of typical experiments}

403 The remaining costs need to be calculated for a typical experiment. We have considered 404 experiments with 1,700 microplots in the field or with 1,700 plants in a robotized platform, 405 together with the costs for plant handling as described in section 2.2, image capture in section 4062.4 , plus the costs of image analysis, data analysis and data storage presented below.

407 2.5.1 Image analysis: a tradeoff between investment in automated workflows and day-to-day 408 labor costs.

409 With the advances in computer vision algorithms and machine learning based classification 410 methods $[5,64,65]$, many image analysis tasks can be accomplished automatically in a high- 
411 throughput fashion. A tradeoff therefore exists between the time dedicated to the development

412 of imaging pipelines and that dedicated to day-to-day image analysis. Several public packages are

413 under development and will hopefully relieve the bottleneck of image analysis [66-68]. This is

414 already largely the case in automated phenotyping platforms, in which routine traits (e.g. plant

415 volume, area or height) are extracted automatically in real time [69-71]; however, sound

416 automatic workflows remain to be required for image series acquired by UAVs or ground

417 vehicles. In both cases, the design of a specific pipeline can result in a cost of nearly \$250-500K

418 USD, if the pipeline is aimed at being sufficiently flexible for different types of users. Much

419 cheaper data acquisition tools are commercially available, designed by companies or plant

420 research laboratories. However, they are often proprietary, designed for specific requests and

421 hence not flexible enough for wider applications. An interesting alternative is that public

422 consortia develop and release flexible analytic workflows, which can then be used and 423 continuously developed by the scientific community through an 'open science and open source'

424 approach. This is currently carried out by different consortia.

425 A cost tradeoff also exists between the quality of images and the time for image analysis. For 426 example, if a standard imaging protocol has not been properly conveyed to end-users (e.g. how 427 to ensure lighting condition and image clarity, how to minimize color distortion, and how to select 428 regions of interest), extra computational work is required to improve the quality of raw data 429 captured by low-cost devices, different formats of raw data might require ongoing licensing or

430 extra fees to carry out trait analysis as well as continued maintenance for future references.

431 The costs in Table 2 are based on the hypothesis of existing workflows and therefore do not 432 consider the cost of their development. With this hypothesis, they still represent $10-20 \%$ of the 433 cost of image capture. As stated above, this cost increases by hundreds of thousands of dollars if 434 the cost of developing workflows is taken into account. It is also considerably higher if image 435 analysis is performed manually. 
438 The datasets resulting from phenotyping projects are difficult to analyze because they are voluminous, complex, heterogeneous, plagued with errors and only can be handled with up-to440 date scientific and mathematical tools. For example, a recent project (EU DROPS) required four 441 full-time PhD students, engineers or post-docs, three technicians and two permanent scientists 442 for four years to conduct data analysis related tasks. This involved compiling and cleaning the datasets collected in fields and greenhouse experiments, designing novel tools for extracting 444 traits from the raw data, and performing cross-scale analyses and genetic analysis. Overall, this 445 procedure recorded a cost of about half a million dollars, i.e. about the same amount dedicated 446 to image analysis in the hypotheses of Table 2.

447 A tradeoff exists between the time dedicated for data capture and analysis. Currently, many 448 phenotyping projects rely on analytic software solutions that are either customized for specific 449 hardware or based on proprietary or specialized software solutions. Similarly, data collected with 450 cost-effective phenotyping approaches are often analyzed manually, which is time consuming,

451 prone to errors and expensive due to additional human costs. Developing workflows with a 452 reproducible data analysis strategy therefore corresponds to a high extra-cost for individual 453 experiments, but it can be considered as a good investment at the level of a broader scientific community, because, in this way, data can be shared, re-used and re-analyzed.

455 Overall, the cost of data analysis is the most underestimated part of many phenotyping projects. 456 In the same way as for image analysis, data analysis costs presented in Table 2 are based on the 457 availability of existing workflows. They considerably increase if workflows need to be developed 458 during the projects, or the whole analysis is performed manually. Based on these hypotheses, 459 the costs required for estimating trait values are similar to those of image analysis. Together, 460 costs of image and data analysis represent $30-200 \%$ of the cost of image capture, a factor that is 461 rarely considered for the overall costs of phenotyping. datasets 
465 The datasets collected above carry more information than any group can handle alone. It is therefore vital for the plant science community to ensure that datasets can be managed in a way that they can be accessed and re-analyzed by scientists that have not been involved in the data collection. By doing that, researchers should be able to trace the history of plants, re-analyze sensor- and image-based datasets with existing or new methods and check sensors in case of inconsistencies. This requires information systems capable of collecting, managing, and presenting thousands of data points and images collected in multiple experiments, together with necessary metadata (FAIR standard: findable, accessible, interoperable and reusable). Such information systems are based on elaborate protocols to describe content and format of phenotypic information [56,72], as well as a standardized description of all involved objects (i.e. plants, organs, sensors, phenotyping facilities) via ontologies $[73,74]$.

The cost for elaborating such information systems involves tens of person-months of computer scientists. As stated in earlier paragraphs, this requires an effort at the level of international consortium. The costs in the hypotheses of Table 2 are based on a pre-existing information system and only consider the cost of data storage (\$32 USD per terabyte per year).

\section{An unexpected structure of costs has large consequences on conclusions about} cost effectiveness

An overall inspection of Table 2 results in a view of phenotyping costs that largely differs from an initial intuition that one might have. In the hypotheses considered in Table 2:

- The cost for handling microplots or plants is by far the highest and is similar in the field and in robotized platforms. The former was based on current costs in most breeding companies; the latter was considered the cost of the greenhouse and of the robot. The cost of microplot or plant handling represents $65-77 \%$ of the total cost of phenotyping, across types of vehicles, hypotheses or location of experiments in a field or a robotized greenhouse.

- The labor cost represents a large proportion of the total cost, from $30 \%$ to $100 \%$ of the cost of vehicles and sensors for data analysis, plus the costs associated with image capture itself. As stated above, these costs are under-estimated in Table 2, because they assume that pipelines already exist for image analysis, trait measurements and associated information systems. These 
costs would considerably increase if the development of pipelines was taken into account, or if all the data processing was considered as manually accomplished.

- Investment itself represents only $10-20 \%$ of the total of phenotyping costs, whereas most discussions on costs focus on investment.

Hence, phenotyping may be one of the few cases in which intuition about cost-effectiveness is not appropriate because (i) it tends to considerably under-estimate personnel and structural costs, (ii) it may lead to choosing tools that are immediately usable and relatively affordable; however, the examples shown previously indicate that heavier investment could result in a more efficient chain for extracting meaningful information.

Other non-intuitive facts also emerge from Table 2 through comparing experiments in robotized platforms in the greenhouse or in the field with imaging based on different vehicles. First, the costs of experiments in a robotized platform are similar to those in the field (Table 2). Second, the total costs of phenotyping do not greatly differ with the choice of vehicles in field experiments. As discussed above, the optimum choice in terms of cost depends on scenarios: for a high demand of phenotyping, the three vehicles result in similar costs, with a slightly lower cost for UAVs; costs increase with a lower demand for the three vehicles, with a slight cost advantage for hand-held ground vehicles. However, these differences are small and context dependent, so a pure cost analysis does not result in an obvious choice between field and platform experiments, or for one of the three considered vehicles.

Overall, the above shows that the cost of phenotyping experiment is high if all related costs are considered (Table 2). However, this statement needs to be contextualized. In some cases, light phenotyping represents a small marginal cost of an operation or experiment that is carried out, for instance, when a farmer needs to take an adequate decision or a breeder needs to keep track of some simple operations, the cost of crop management is not considered and the need for data analysis and storage is limited. Mobile phones or inexpensive UAV flights for light phenotyping are therefore highly valuable in these cases. In the other extreme, a phenotyping project aiming to characterize hundreds of genotypes needs to take all costs into account, resulting in a high overall cost for plant handling together with a high manpower cost for data analysis and data storage. Investment in vehicles and imaging devices therefore represent a limited proportion of 
522 the total cost. In this case, the choice of vehicle (UAV vs ground vehicle), location, and 523 experiments (field vs platform) should be taken into consideration together with other factors, 524 i.e. the nature and the precision of the desired traits as well as the constraints linked to the management of personnel.

526 Numerous trade-offs have been presented here between investment and operational costs, for 527 example, the choice of vehicles, imaging techniques, or image analysis workflows. Hence, 528 'affordable phenotyping', considered as the way to obtain a maximum of images in a minimum 529 time frame with low investment costs, may be counter-productive in many cases. Similarly, the 530 development of analysis pipelines represents a large investment but often lead to cheaper and 531 more reproducible datasets than manual or tailored analysis in the long term. These trade-offs 532 depend on local conditions, such as the availability, the cost of manpower, and the number of 533 days, during which a given device can be used per year due to climatic or other constraints. None 534 of the devices or techniques discussed above can be considered as cost-effective or cost535 ineffective per se, as nearly all of them can be considered adequate for specific tasks under 536 defined conditions and ineffective in other circumstances.

537 It is therefore essential that costs are reasoned in relation to (i) the precision, repeatability and 538 heritability required in a given phenotyping task (ii) local personnel costs (training, data transfer, 539 data calibration, data analysis and data management) that greatly vary between projects and 540 countries, (iii) the cost per unit plot or trait, which can largely differ between methods depending 541 on local climatic and economic conditions. If all of these elements are taken into account, 'cost 542 effective' phenotyping may in some cases involve low investment ('affordable phenotyping'), and 543 in other cases involve an initial high investment that results in low operational costs together 544 with high quality outcomes. Finally, for breeding purpose, phenotyping costs also need to be 545 analyzed in terms of their contribution to the rate of genetic gain. Direct ratios cannot be established at this stage because of uncertainties about the scalability of measured traits towards 547 yield in the absence of case studies combining phenomics, modelling and genomic prediction 548 [75]. However, one piece of equipment and associated methods in phenomics have already 549 shown their contribution to breeding: it has been observed here that the investment in sensor 550 networks for environmental characterization has a clear value for interpretation of the genotype 
$551 \mathrm{x}$ environment interaction, and for weighing the investment in specific breeding programs in

552 relation to the frequency of corresponding target populations of environments.

553

554 Acknowledgements

555 Authors are grateful to the phenotyping communities in the French and UK phenotyping 556 infrastructures for cost evaluation. FT, CW and FB thank the ANR-PIA project PHENOME FPPN 557 (ANR-11-INBS-0012) and EPPN ${ }^{2020}$ (UE H2020 grant agreement No 731013) for partly funding this 558 work. JZ, DR, AB and JB are funded by the Biotechnology and Biological Sciences Research Council 559 (BBSRC), Core Strategic Programme Grant (BB/CSP17270/1) at EI, BBSRC's Designing Future 560 Wheat Strategic Programme (BB/P016855/1) to G. Moore and (BBS/E/T/000PR9785) to JZ.

\section{References}

563 [1] F. Tardieu, L. Cabrera-Bosquet, T. Pridmore, M. Bennett, Plant Phenomics, From Sensors to Knowledge, Curr. Biol. 27 (2017) R770-R783. doi:10.1016/j.cub.2017.05.055.

[2] R.T. Furbank, M. Tester, Phenomics--technologies to relieve the phenotyping bottleneck., Trends Plant Sci. 16 (2011) 635-44. doi:10.1016/j.tplants.2011.09.005.

[3] F. Fiorani, U. Schurr, Future scenarios for plant phenotyping., Annu. Rev. Plant Biol. 64 (2013) 267-91. doi:10.1146/annurev-arplant-050312-120137.

[4] D. Deery, J. Jimenez-Berni, H. Jones, X. Sirault, R. Furbank, Proximal Remote Sensing Buggies and Potential Applications for Field-Based Phenotyping, 2014.

572 [5] D. Rousseau, H. Dee, T. Pridmore, Imaging Methods for Phenotyping of Plant Traits, in: S. Kumar (Ed.), Phenomics Crop Plants Trends, Options Limitations, Springer India, 2015: pp. 61-74. doi:DOI 10.1007/978-81-322-2226-2_5.

[6] L. Busemeyer, D. Mentrup, K. Möller, E. Wunder, K. Alheit, V. Hahn, H.P. Maurer, J.C. Reif, T. Würschum, J. Müller, F. Rahe, A. Ruckelshausen, Breedvision - A multi-sensor 
platform for non-destructive field-based phenotyping in plant breeding, Sensors (Switzerland). 13 (2013) 2830-2847. doi:10.3390/s130302830.

[7] G. Bitella, R. Rossi, R. Bochicchio, M. Perniola, M. Amato, A novel low-cost openhardware platform for monitoring soil water content and multiple soil-air-vegetation parameters, Sensors (Switzerland). 14 (2014) 19639-19659. doi:10.3390/s141019639.

[8] M.J. Aitkenhead, G.J. Gaskin, N. Lafouge, C. Hawes, Phylis: A low-cost portable visible range spectrometer for soil and plants, Sensors (Switzerland). 17 (2017). doi:10.3390/s17010099.

[9] A. Pask, J. Pietragalla, D. Mullan, M. Reynolds, Physiological Breeding II: A Field Guide to Wheat Phenotyping, CIMMYT, Mexico, 2012. doi:10.1017/СBO9781107415324.004.

[10] J. Barker, N. Zhang, J. Sharon, R. Steeves, X. Wang, Y. Wei, J. Poland, Development of a field-based high-throughput mobile phenotyping platform, Comput. Electron. Agric. 122 (2016) 74-85. doi:10.1016/j.compag.2016.01.017.

[11] H.M. Easlon, A.J. Bloom, Easy Leaf Area: Automated Digital Image Analysis for Rapid and Accurate Measurement of Leaf Area, Appl. Plant Sci. 2 (2014) 1400033. doi:10.3732/apps.1400033.

[12] D. Kelly, A. Vatsa, W. Mayham, L. Ng, A. Thompson, T. Kazic, An opinion on imaging challenges in phenotyping field crops, Mach. Vis. Appl. 27 (2016) 681-694. doi:10.1007/s00138-015-0728-4.

[13] Y. Intaravannea, S. Sumriddetchkajorn, Android-based rice leaf color analyzer for estimating the needed amount of nitrogen fertilizer, Comput. Electron. Agric. 116 (2015) 228-233. doi:https://doi.org/10.1016/j.compag.2015.07.005.

[14] E. Komyshev, M. Genaev, D. Afonnikov, Evaluation of the SeedCounter, A Mobile Application for Grain Phenotyping, Front. Plant Sci. 7 (2017) 1-9. doi:10.3389/fpls.2016.01990.

[15] R. Confalonieri, M. Foi, R. Casa, S. Aquaro, E. Tona, M. Peterle, A. Boldini, G. De Carli, A. Ferrari, G. Finotto, T. Guarneri, V. Manzoni, E. Movedi, A. Nisoli, L. Paleari, I. Radici, M. 
Suardi, D. Veronesi, S. Bregaglio, G. Cappelli, M.E. Chiodini, P. Dominoni, C. Francone, N. Frasso, T. Stella, M. Acutis, Development of an app for estimating leaf area index using a smartphone. Trueness and precision determination and comparison with other indirect methods, Comput. Electron. Agric. 96 (2013) 67-74. doi:10.1016/J.COMPAG.2013.04.019.

[16] A. Burkart, V.L. Hecht, T. Kraska, R. U., Phenological analysis of unmanned aerial vehicle based time series of barley imagery with high temporal resolution, Precis. Agric. (2017) 1-13. doi:oi.org/10.1007/s11119-017-9504-y.

[17] S. Sankaran, L.R. Khot, C.Z. Espinoza, S. Jarolmasjed, V.R. Sathuvalli, G.J. Vandemark, P.N. Miklas, A.H. Carter, M.O. Pumphrey, R.R.N. Knowles, M.J. Pavek, Low-altitude, highresolution aerial imaging systems for row and field crop phenotyping: A review, Eur. J. Agron. 70 (2015) 112-123. doi:10.1016/j.eja.2015.07.004.

[18] T. Duan, B. Zheng, W. Guo, S. Ninomiya, Y. Guo, S.C. Chapman, T.D. A, B.Z. A, W.G. C, S.N. C, Y.G. B, S.C.C. A, Comparison of ground cover estimates from experiment plots in cotton, sorghum and sugarcane based on images and ortho-mosaics captured by UAV, Funct. Plant Biol. (2016). doi:10.1071/FP16123.

[19] N. Brisson, P. Gate, D. Gouache, G. Charmet, F.X. Oury, F. Huard, Why are wheat yields stagnating in Europe? A comprehensive data analysis for France, F. Crop. Res. 119 (2010) 201-212. doi:10.1016/j.fcr.2010.07.012.

[20] V.O. Sadras, R.A. Richards, Improvement of crop yield in dry environments: Benchmarks, levels of organisation and the role of nitrogen, J. Exp. Bot. 65 (2014) 1981-1995. doi:10.1093/jxb/eru061.

[21] M. Cooper, F.A. van Eeuwijk, G.L. Hammer, D.W. Podlich, C. Messina, Modeling QTL for complex traits: detection and context for plant breeding, Curr. Opin. Plant Biol. 12 (2009) 231-240. doi:10.1016/j.pbi.2009.01.006.

[22] J. Zhou, C. Applegate, A.D. Alonso, D. Reynolds, S. Orford, M. Mackiewicz, S. Griffiths, S. Penfield, N. Pullen, Leaf-GP: An Open and Automated Software Application for 
Measuring Growth Phenotypes for Arabidopsis and Wheat, Plant Methods. 13 (2017) 130. doi:https://doi.org/10.1101/180083.

[23] W. Guo, T. Fukatsu, S. Ninomiya, Automated characterization of flowering dynamics in rice using field-acquired time-series RGB images, Plant Methods. 11 (2015). doi:10.1186/s13007-015-0047-9.

[24] M. Reynolds, P. Langridge, Physiological breeding, Curr. Opin. Plant Biol. 31 (2016) 162171. doi:10.1016/j.pbi.2016.04.005.

[25] F. Tardieu, T. Simonneau, B. Muller, The Physiological Basis of Drought Tolerance in Crop Plants : A Scenario-Dependent Probabilistic Approach, Annu. Rev. Plant Biol. 69 (2018) 733-759. doi:10.1146/annurev-arplant-042817- 040218.

[26] J.C. hristian Rose, S. Paulus, H. Kuhlmann, Accuracy analysis of a multi-view stereo approach for phenotyping of tomato plants at the organ level, Sensors (Basel). 15 (2015) 9651-9665. doi:10.3390/s150509651.

[27] M. Müller-Linow, F. Pinto-Espinosa, H. Scharr, U. Rascher, The leaf angle distribution of natural plant populations: assessing the canopy with a novel software tool, Plant Methods. 11 (2015) 11. doi:10.1186/s13007-015-0052-z.

[28] R. Khanna, J. Rehder, M. Martin, E. Galceran, R. Siegwart, Studying Phenotypic Variability in Crops using a Hand-held Sensor Platform, IROS Work. Agri-Food Robot. (2015).

[29] J.L. Crain, Y. Wei, J. Barker, S.M. Thompson, P.D. Alderman, M. Reynolds, N. Zhang, J. Poland, Development and deployment of a portable field phenotyping platform, Crop Sci. 56 (2016) 965-975. doi:10.2135/cropsci2015.05.0290.

[30] S. Kuhlgert, G. Austic, R. Zegarac, I. Osei-Bonsu, D. Hoh, M.I. Chilvers, M.G. Roth, K. Bi, D. TerAvest, P. Weebadde, D.M. Kramer, MultispeQ Beta: a tool for large-scale plant phenotyping connected to the open PhotosynQ network., R. Soc. Open Sci. 3 (2016) 160592. doi:10.1098/rsos.160592.

[31] I. Jonckheere, S. Fleck, K. Nackaerts, B. Muys, P. Coppin, M. Weiss, F. Baret, Review of methods for in situ leaf area index determination: Part I. Theories, sensors and 
hemispherical photography, Agric. For. Meteorol. 121 (2004) 19-35.

doi:10.1016/J.AGRFORMET.2003.08.027.

[32] M. Weiss, F. Baret, G.J. Smith, I. Jonckheere, P. Coppin, Review of methods for in situ leaf area index (LAI) determination, part II: Estimation of LAI, errors and sampling., Agric. For. Meteorol. 121 (2004) 37-53. doi:10.1016/j.agrformet.2003.08.001.

[33] J. Berni, P.J. Zarco-Tejada, L. Suarez, E. Fereres, Thermal and Narrowband Multispectral Remote Sensing for Vegetation Monitoring From an Unmanned Aerial Vehicle, IEEE Trans. Geosci. Remote Sens. 47 (2009) 722-738. doi:10.1109/TGRS.2008.2010457.

[34] H.F. Phenotyping, M.F. Dreccer, E. Holland, B. Zheng, T.J. Ling, Pheno-Copter: A LowAltitude, Autonomous Remote-Sensing Robotic Helicopter for High-Throughput FieldBased Phenotyping, Agronomy. 4 (2014) 279-301. doi:10.3390/agronomy4020279.

[35] F. Baret, S. Madec, K. Irfan, J. Lopez, A. Comar, D. Dutartre, S. Praud, M.H. Tixier, Leaf rolling in maize crops : from leaf scoring to canopy level measurements for phenotyping ., BioRxiv. (2017) 1-35.

[36] D.M. Deery, G.J. Rebetzke, J.A. Jimenez-Berni, R.A. James, A.G. Condon, W.D. Bovill, P. Hutchinson, J. Scarrow, R. Davy, R.T. Furbank, Methodology for High-Throughput Field Phenotyping of Canopy Temperature Using Airborne Thermography, Front. Plant Sci. 7 (2016) 1-13. doi:10.3389/fpls.2016.01808.

[37] X. Jin, S. Liu, F. Baret, M. Hemerlé, A. Comar, Estimates of plant density of wheat crops at emergence from very low altitude UAV imagery, Remote Sens. Environ. 198 (2017) 105114. doi:10.1016/J.RSE.2017.06.007.

[38] A. Gracia-Romero, S.C. Kefauver, O. Vergara-Diaz, M.A. Zaman-Allah, B.M. Prasanna, J.E. Cairns, J.L. Araus, Comparative performance of ground versus aerially assessed RGB and multispectral indices for early-growth evaluation of maize performance under phosphorus fertilization I n r e v i e w, Front. Plant Sci. 8 (2017) 1-13. doi:10.3389/fpls.2017.02004.

[39] F.H. Holman, A.B. Riche, A. Michalski, M. Castle, M.J. Wooster, M.J. Hawkesford, High 
throughput field phenotyping of wheat plant height and growth rate in field plot trials using UAV based remote sensing, Remote Sens. 8 (2016). doi:10.3390/rs8121031.

[40] L. Malambo, S.C. Popescu, S.C. Murray, E. Putman, N.A. Pugh, D.W. Horne, G. Richardson, R. Sheridan, W.L. Rooney, R. Avant, M. Vidrine, B. McCutchen, D. Baltensperger, M. Bishop, Multitemporal field-based plant height estimation using 3D point clouds generated from small unmanned aerial systems high-resolution imagery, Int. J. Appl. Earth Obs. Geoinf. 64 (2018) 31-42. doi:10.1016/j.jag.2017.08.014.

[41] K. Watanabe, W. Guo, K. Arai, H. Takanashi, H. Kajiya-Kanegae, M. Kobayashi, K. Yano, T. Tokunaga, T. Fujiwara, N. Tsutsumi, H. Iwata, High-Throughput Phenotyping of Sorghum Plant Height Using an Unmanned Aerial Vehicle and Its Application to Genomic Prediction Modeling, Front. Plant Sci. 8 (2017) 1-11. doi:10.3389/fpls.2017.00421.

[42] W. Li, Z. Niu, H. Chen, D. Li, M. Wu, W. Zhao, Remote estimation of canopy height and aboveground biomass of maize using high-resolution stereo images from a low-cost unmanned aerial vehicle system, Ecol. Indic. 67 (2016) 637-648. doi:10.1016/j.ecolind.2016.03.036.

[43] Y. Lin, LiDAR: An important tool for next-generation phenotyping technology of high potential for plant phenomics?, Comput. Electron. Agric. 119 (2015) 61-73. doi:10.1016/j.compag.2015.10.011.

[44] A. Verger, N. Vigneau, C. Che??ron, J.M. Gilliot, A. Comar, F. Baret, Green area index from an unmanned aerial system over wheat and rapeseed crops, Remote Sens. Environ. 152 (2014) 654-664. doi:10.1016/j.rse.2014.06.006.

[45] M. Ishihara, Y. Inoue, K. Ono, M. Shimizu, S. Matsuura, The impact of sunlight conditions on the consistency of vegetation indices in croplands-Effective usage of vegetation indices from continuous ground-based spectral measurements, Remote Sens. 7 (2015) 14079-14098. doi:10.3390/rs71014079.

[46] B. Stark; , T. Zhao, Y. Chen, An analysis of the effect of the bidirectional reflectance distribution function on remote sensing imagery accuracy from Small Unmanned Aircraft 
Systems, in: 2016 Int. Conf. Unmanned Aircr. Syst., ICUAS, Arlington, VA, USA, 2016. doi:10.1109/ICUAS.2016.7502566.

[47] J.W. White, M.M. Conley, A flexible, low-cost cart for proximal sensing, Crop Sci. 53 (2013) 1646-1649. doi:10.2135/cropsci2013.01.0054.

[48] M.P. Cendrero-Mateo, O. Muller, H. Albrecht, A. Burkart, S. Gatzke, B. Janssen, B. Keller, N. Körber, T. Kraska, S. Matsubara, J. Li, M. Müller-Linow, R. Pieruschka, F. Pinto, P. Rischbeck, A. Schickling, A. Steier, M. Watt, U. Schurr, U. Rascher, Field Phenotyping: Challenges and Opportunities, Terr. Ecosyst. Res. Infrastructures. (2017) 53-80. doi:10.1201/9781315368252-4.

[49] J. Svensgaard, T. Roitsch, S. Christensen, Development of a Mobile Multispectral Imaging Platform for Precise Field Phenotyping, Agronomy. 4 (2014) 322-336. doi:10.3390/agronomy4030322.

[50] K. Jensen, S.H. Nielsen, R.N. Jorgensen, A. Bogild, N.J. Jacobsen, O.J. Jorgensen, C.L. Jaeger-Hansen, A LOW COST, MODULAR ROBOTICS TOOL CARRIER FOR PRECISION AGRICULTURE RESEARCH K. Jensen, S.H. Nielsen, and R.N. Jørgensen, Int. Conf. Precis. Agric. (2012).

[51] L. Grimstad, P. From, The Thorvald II Agricultural Robotic System, Robotics. 6 (2017) 24. doi:10.3390/robotics6040024.

[52] A. Shafiekhani, S. Kadam, F.B. Fritschi, G.N. Desouza, Vinobot and vinoculer: Two robotic platforms for high-throughput field phenotyping, Sensors. 17 (2017) 1-23. doi:10.3390/s17010214.

[53] E. Millet, C. Welcker, W. Kruijer, S. Negro, S. Nicolas, S. Praud, N. Ranc, T. Presterl, R. Tuberosa, Z. Bedo, X. Draye, B. Usadel, A. Charcosset, F. van Eeuwijk, F. Tardieu, A. Coupel-Ledru, C. Bauland, Genome-wide analysis of yield in Europe: allelic effects as functions of drought and heat scenarios, Plant Physiol. 172 (2016) pp.00621.2016. doi:10.1104/pp.16.00621.

[54] L. Cabrera-Bosquet, C. Fournier, N. Brichet, C. Welcker, B. Suard, F. Tardieu, High- 
throughput estimation of incident light, light interception and radiation-use efficiency of thousands of plants in a phenotyping platform, New Phytol. 212 (2016) 269-281. doi:10.1111/nph.14027.

[55] G. Villarrubia, J.F. De Paz, D.H. De La Iglesia, J. Bajo, Combining multi-agent systems and wireless sensor networks for monitoring crop irrigation, Sensors (Switzerland). 17 (2017). doi:10.3390/s17081775.

[56] J. Zhou, D. Reynolds, D. Websdale, T. Le Cornu, O. Gonzalez-Navarro, C. Lister, S. Orford, S. Laycock, G. Finlayson, T. Stitt, M. Clark, M. Bevan, S. Griffiths, CropQuant: An automated and scalable field phenotyping platform for crop monitoring and trait measurements to facilitate breeding and digital agriculture, BioRxiv. (2017) 1-17. doi:https://doi.org/10.1101/161547.

[57] K. Chenu, M. Cooper, G.L. Hammer, K.L. Mathews, M.F. Dreccer, S.C. Chapman, Environment characterization as an aid to wheat improvement: Interpreting genotypeenvironment interactions by modelling water-deficit patterns in North-Eastern Australia, J. Exp. Bot. 62 (2011) 1743-1755. doi:10.1093/jxb/erq459.

[58] M.T. Harrison, F. Tardieu, Z. Dong, C.D. Messina, G.L. Hammer, Characterizing drought stress and trait influence on maize yield under current and future conditions, Glob. Chang. Biol. 20 (2014) 867-878. doi:10.1111/gcb.12381.

[59] Y. Xu, Envirotyping for deciphering environmental impacts on crop plants, Theor. Appl. Genet. 129 (2016) 653-673. doi:10.1007/s00122-016-2691-5.

[60] M. Cooper, C. Gho, R. Leafgren, T. Tang, C. Messina, Breeding drought-tolerant maize hybrids for the US corn-belt: Discovery to product, J. Exp. Bot. 65 (2014) 6191-6194. doi:10.1093/jxb/eru064.

[61] A. Murigneux, F. HENRIOT, M. PERSONNE, M. RENAULT, C. DELLUC, R. DEBEUF, Method for determining drought tolerance in maize, PCT/EP2016/063067, 2015. https://patents.google.com/patent/WO2016198471A1/en.

[62] V. Vadez, J. Kholová, G. Hummel, U. Zhokhavets, S.K. Gupta, C.T. Hash, LeasyScan: A 
novel concept combining 3D imaging and lysimetry for high-throughput phenotyping of traits controlling plant water budget, J. Exp. Bot. 66 (2015) 5581-5593. doi:10.1093/jxb/erv251.

[63] S. Lacube, C. Fournier, C. Palaffre, E.J. Millet, F. Tardieu, B. Parent, Distinct controls of leaf widening and elongation by light and evaporative demand in maize, Plant Cell Environ. 40 (2017) 2017-2028.

[64] S. van der Walt, J.L. Schönberger, J. Nunez-Iglesias, F. Boulogne, J.D. Warner, N. Yager, E. Gouillart, T. Yu, Scikit-image: image processing in Python., PeerJ. 2 (2014) 1-18. doi:10.7717/peerj.453.

[65] J. Howse, OpenCV Computer Vision with Python, 1st ed., Packt Publishing Ltd., Birmingham, UK, 2013.

[66] A. Singh, B. Ganapathysubramanian, A.K. Singh, S. Sarkar, Machine Learning for HighThroughput Stress Phenotyping in Plants, Trends Plant Sci. 21 (2016) 110-124. doi:10.1016/j.tplants.2015.10.015.

[67] M.P. Pound, J.A. Atkinson, A.J. Townsend, M.H. Wilson, M. Griffiths, A.S. Jackson, A. Bulat, G. Tzimiropoulos, D.M. Wells, E.H. Murchie, T.P. Pridmore, A.P. French, Deep machine learning provides state-of-the-art performance in image-based plant phenotyping, Gigascience. 6 (2017) 1-10. doi:10.1093/gigascience/gix083.

[68] M.P. Pound, J.A. Atkinson, D.M. Wells, T.P. Pridmore, A.P. French, Deep Learning for Multi-task Plant Phenotyping, in: IEEE Int. Conf. Comput. Vis., 2017: pp. 2055-2063. doi:10.1101/204552.

[69] C. Klukas, D. Chen, J.-M. Pape, Integrated Analysis Platform: An Open-Source Information System for High-Throughput Plant Phenotyping, Plant Physiol. 165 (2014) 506-518. doi:10.1104/pp.113.233932.

[70] M.J. Hawkesford, A. Lorence, Plant phenotyping : increasing throughput and precision at multiple scales, Funct. Plant Biol. (2017). doi:10.1071/FP.

[71] N. Kirchgessner, F. Liebisch, K. Yu, J. Pfeifer, M. Friedli, A. Hund, A. Walter, The ETH field 
phenotyping platform FIP: A cable-suspended multi-sensor system, Funct. Plant Biol. 44 (2017) 154-168. doi:10.1071/FP16165.

[72] C. Pradal, S. Artzet, J. Chopard, D. Dupuis, C. Fournier, M. Mielewczik, V. Nègre, P. Neveu, D. Parigot, P. Valduriez, S. Cohen-Boulakia, InfraPhenoGrid: A scientific workflow infrastructure for plant phenomics on the Grid, Futur. Gener. Comput. Syst. 67 (2017) 341-353. doi:10.1016/j.future.2016.06.002.

[73] Y.F. Li, G. Kennedy, F. Ngoran, P. Wu, J. Hunter, An ontology-centric architecture for extensible scientific data management systems, Futur. Gener. Comput. Syst. 29 (2013) 641-653. doi:10.1016/j.future.2011.06.007.

[74] H. Ćwiek-Kupczyńska, T. Altmann, D. Arend, E. Arnaud, D. Chen, G. Cornut, F. Fiorani, W. Frohmberg, A. Junker, C. Klukas, M. Lange, C. Mazurek, A. Nafissi, P. Neveu, J. van Oeveren, C. Pommier, H. Poorter, P. Rocca-Serra, S.-A. Sansone, U. Scholz, M. van Schriek, Ü. Seren, B. Usadel, S. Weise, P. Kersey, P. Krajewski, Measures for interoperability of phenotypic data: minimum information requirements and formatting, Plant Methods. 12 (2016) 44. doi:10.1186/s13007-016-0144-4.

[75] J. Crain, S. Mondal, J. Rutkoski, R.P. Singh, J. Poland, Combining High-Throughput Phenotyping and Genomic Information to Increase Prediction and Selection Accuracy in Wheat Breeding, Plant Genome. 11 (2018) 0. doi:10.3835/plantgenome2017.05.0043. 
813 Table 1. Imaging costs involving vehicle, sensors, associated software and personnel in field experiments or in a robotized platform,

814 for two scenarios of demand for phenotyping (offer or demand-limited) and, in the field, three categories of vehicles (vectors) carrying

815 sensors (automated or hand-held ground vehicle or unmanned aerial vehicle (UAV). Costs are expressed in US dollars per plot.day per

816 year (field) or plant.day per year (robotized platform), with the principles of calculations in the panel "vector". Costs of manpower are

817 calculated per year and plot.day or plant.day. Two scenarios are considered for field conditions: in scenario 1 (offer limited), the

818 demand for phenotyping exceeds the capacity of the system; in scenario 2 (demand limited) the demand represents a maximum of

8194000 microplots per year.

\begin{tabular}{|c|c|c|c|c|c|c|c|c|c|c|c|c|}
\hline & & & & Vector & & & Sensors & Manpou & + training & $\mathrm{Ma}$ & enance & Cost imaging \\
\hline & $\begin{array}{l}\text { Hypotheses for } \\
\text { each scenario }\end{array}$ & $\begin{array}{c}\text { Days of use } \\
\text { year }^{-1}\end{array}$ & $\begin{array}{l}\text { Throughput, } \\
\text { uplot or } \\
\text { plant day }{ }^{-1}\end{array}$ & $\begin{array}{l}\text { Expected } \\
\text { duration, } \\
\text { year }\end{array}$ & $\begin{array}{c}\text { Investment } \\
\mathrm{k} \$\end{array}$ & $\begin{array}{c}\text { Investment } \\
\$ \text { per plot } \\
\text { per day } \\
\text { vector life } \\
\end{array}$ & $\begin{array}{l}\text { Equivalent } \\
\text { calculation, } \\
4 \text { year life }\end{array}$ & $\$$ year $^{-1}$ & $\begin{array}{l}\text { per plot } \\
\text { day per } \\
\text { year }\end{array}$ & $\$$ year $^{-1}$ & $\begin{array}{l}\text { \$per plot } \\
\text { day.plot } \\
\text { peryear }\end{array}$ & $\begin{array}{c}\text { \$ per plot } \\
\text { day per year }\end{array}$ \\
\hline $\begin{array}{l}\text { High throughput } \\
\text { field experiments, 'offer } \\
\text { limited' }\end{array}$ & $\begin{array}{l}\text { Limited by availability } \\
\text { of equipment and } \\
\text { personnel. }\end{array}$ & & & & & & & & & & & \\
\hline Automated ground vehicle & & 60 & 1200 & 20 & 430 & 0.30 & 0.24 & 19564 & 0.2717 & 15000 & 0.2083 & 1.02 \\
\hline Hand-held ground vehicle & & 50 & 800 & 15 & 50 & 0.08 & 0.44 & 15553 & 0.3888 & 3000 & 0.0750 & 0.98 \\
\hline UAV & & 40 & 4000 & 2 & 10 & 0.03 & 0.09 & 24545 & 0.1534 & 2000 & 0.0125 & 0.29 \\
\hline $\begin{array}{l}\text { High throughputfield } \\
\text { experiments, 'demand } \\
\text { limited' }\end{array}$ & $\begin{array}{l}\text { Limited by the demand } \\
\text { for microplot per year. } \\
40000 \text { pplots year }{ }^{-1}\end{array}$ & & & & & & & & & & & \\
\hline Automated ground vehicle & & 33 & 1200 & 20 & 430 & 0.54 & 0.44 & 12873 & 0.3218 & 15000 & 0.3750 & 1.67 \\
\hline Hand-held ground vehicle & & 50 & 800 & 15 & 50 & 0.08 & 0.44 & 15553 & 0.3888 & 3000 & 0.0750 & 0.98 \\
\hline UAV & & 10 & 4000 & 2 & 10 & 0.13 & 0.38 & 17018 & 0.4255 & 2000 & 0.0500 & 0.98 \\
\hline Robotized indoor platform & $\begin{array}{l}\text { Limited by availability } \\
\text { of equipment and } \\
\text { personnel. }\end{array}$ & 270 & 1700 & 15 & 1000 & 0.15 & 0.02 & 103618 & 0.2257 & 15000 & 0.0327 & 0.42 \\
\hline
\end{tabular}

820

personnel. 
822 Table 2 Distribution of costs in typical experiments in the field (1,700 microplots with 10 days of observation) or robotized platforms 823 (1700 plants with 90 days). Hypotheses are as above. The cost of microplot or plant handling represents either the current costs per 824 plot (field) or the cost of greenhouse plus robot, together with manpower (robotized platform). Note that the cost of the robot was 825 considered in "investment" in Table 1 but is in "plant handling" in Table 2 for easier comparison with the field. Robots are used for both plant handling and imaging in robotized platforms. 'Meas' stands for 'measurements'

\begin{tabular}{|c|c|c|c|c|c|c|c|c|}
\hline & & $\begin{array}{c}\text { Cost } \mu \text { plots } \\
\text { or plant } \\
\text { handling } \\
\mathrm{k} \$\end{array}$ & $\begin{array}{c}\text { Image } \\
\text { capture } \\
\text { k\$ }\end{array}$ & $\begin{array}{c}\text { Image } \\
\text { analysis } \\
\mathrm{k} \$\end{array}$ & $\begin{array}{c}\text { Trait } \\
\text { analysis } \\
\text { k\$ }\end{array}$ & $\begin{array}{c}\text { Data } \\
\text { storage } \\
10 \text { years, k\$ }\end{array}$ & $\begin{array}{c}\text { Total } \\
\mathbf{k} \$\end{array}$ & $\begin{array}{c}\% \\
\text { investment }\end{array}$ \\
\hline \multicolumn{9}{|l|}{$\begin{array}{l}\text { High throughput } \\
\text { field experiments, 'offer } \\
\text { limited' }\end{array}$} \\
\hline Automated ground vehicle & 1700 uplots, 10 days meas & 68.0 & 17.4 & 3.5 & 5.3 & 1.5 & 96 & 18.2 \\
\hline Hand-held ground vehicle & 1700 uplots, 10 days meas & 68.0 & 16.7 & 5.3 & 7.1 & 0.7 & 98 & 17.1 \\
\hline UAV & 1700 uplots, 10 days meas & 68.0 & 4.9 & 7.1 & 10.6 & 0.2 & 91 & 5.4 \\
\hline \multicolumn{9}{|l|}{$\begin{array}{l}\text { High throughputfield } \\
\text { experiments, 'demand } \\
\text { limited' }\end{array}$} \\
\hline Automated ground vehicle & 1700 pplots, 10 days meas & 68.0 & 28.4 & 3.5 & 5.3 & 1.5 & 107 & 26.6 \\
\hline Hand-held ground vehicle & 1700 uplots, 10 days meas & 68.0 & 16.7 & 5.3 & 7.1 & 0.7 & 98 & 17.1 \\
\hline UAV & 1700 pplots, 10 days meas & 68.0 & 16.6 & 7.1 & 10.6 & 0.2 & 103 & 16.2 \\
\hline $\begin{array}{l}\text { Robotized platform } \\
\text { the cost of robot is in the } \\
\text { 'handling' column }\end{array}$ & 1700 plants, 90 days & 71.2 & 9.0 & 1.8 & 10.6 & 2.6 & 95 & 9.5 \\
\hline
\end{tabular}


